PROCEEDINGS OF THE

AMERICAN MATHEMATICAL SOCIETY

Volume 136, Number 9, September 2008, Pages 3185-3193

S 0002-9939(08)09299-X

Article electronically published on April 29, 2008

\title{
SELF-COMMUTATORS OF AUTOMORPHIC COMPOSITION OPERATORS ON THE DIRICHLET SPACE
}

\author{
A. ABDOLLAHI
}

(Communicated by N. Tomczak-Jaegermann)

\begin{abstract}
Let $\varphi$ be a conformal automorphism on the unit disk $\mathbb{U}$ and $C_{\varphi}: \mathcal{D} \longrightarrow \mathcal{D}$ be the composition operator on the Dirichlet space $\mathcal{D}$ induced by $\varphi$. In this article we completely determine the point spectrum, spectrum, essential spectrum and essential norm of the operators $C_{\varphi}^{*} C_{\varphi}, C_{\varphi} C_{\varphi}^{*}$ and self-commutators of $C_{\varphi}$, which expose that the spectrum and point spectrum coincide. We also find the eigenfunctions of the operators.
\end{abstract}

\section{INTRODUCTION}

Let $\varphi$ be a holomorphic self-map of the unit disk $\mathbb{U}:=\{z \in \mathbb{C}:|z|<1\}$. The function $\varphi$ induces the composition operator $C_{\varphi}$, defined on the space of holomorphic functions on $\mathbb{U}$ by $C_{\varphi} f=f \circ \varphi$. The restriction of $C_{\varphi}$ to various Banach spaces of holomorphic functions on $\mathbb{U}$ has been an active subject of research for more than three decades, and it will continue to be for decades to come (see [15, [16] and [5]). Let $\mathcal{D}$ denote the Dirichlet space of analytic functions on the unit disk with derivatives that are square integrable with respect to the area measure on the disk. In recent years the study of composition operators on the the Dirichlet space has received considerable attention (see [6, [3, 7], 10, 11] and the references cited therein).

The main aim here is to find the spectrum, point spectrum, essential spectrum and essential norm of $C_{\varphi}^{*} C_{\varphi}, C_{\varphi} C_{\varphi}^{*}$, self-commutator $\left[C_{\varphi}^{*}, C_{\varphi}\right]=C_{\varphi}^{*} C_{\varphi}-C_{\varphi} C_{\varphi}^{*}$ and anti-self-commutator $\left\{C_{\varphi}^{*}, C_{\varphi}\right\}=C_{\varphi}^{*} C_{\varphi}+C_{\varphi} C_{\varphi}^{*}$, for automorphic composition operators $C_{\varphi}$ on the Dirichlet space.

Actually, this work is an extension of the work of an earlier paper by P. S. Bourdon and B. MacCluer 2 to the Dirichlet space. In [2], by using Cowen's formula for the adjoint of $C_{\varphi}$ on $H^{2}(\mathbb{U})$, the authors have completely determined the spectrum, essential spectrum and point spectrum for self-commutators of automorphic composition operators acting on the Hardy space of unit disk. By using Cowen's formula extensions to $A^{2}\left(B_{N}\right)$ and $H^{2}\left(B_{N}\right)$ for $N \geq 1$, they have also shown that, for each

Received by the editors May 14, 2007, and, in revised form, July 16, 2007.

2000 Mathematics Subject Classification. Primary 47B33; Secondary 47A10, 47E20, $47 \mathrm{~B} 47$.

Key words and phrases. Dirichlet space, composition operator, spectrum, essential spectrum, essential norm, self-commutator, anti-self-commutator.

This research was partially supported by a grant from the Shiraz University Research Council.

This work was carried out at the Department of Mathematics, University of Auckland, where the author was on the sabbatical leave during the academic year 2006-2007.

(C)2008 American Mathematical Society 3185

Reverts to public domain 28 years from publication 
automorphic composition operator $C_{\varphi}$ acting on these spaces, $C_{\varphi}^{*} C_{\varphi}$ is a Toeplitz operator and $C_{\varphi} C_{\varphi}^{*}$ is the inverse of a Toeplitz operator, and the self-commutator $\left[C_{\varphi}^{*}, C_{\varphi}\right]$ is essentially a Toeplitz operator. They have a complete description of the spectrum and essential spectrum of $\left[C_{\varphi}^{*}, C_{\varphi}\right]$, acting on the Hardy and Bergman spaces, when $\varphi$ is an involution automorphism, i.e., when $\varphi \circ \varphi=I$. In this case, as an application of the results in the paper, they have determined the shape of the numerical range of $C_{\varphi}$, which was determined in [1] by using matrix methods.

The other problem which is important to the study of composition operators is finding the relationships between the properties of the symbol $\varphi$ and essential normality of the composition operator $C_{\varphi}$. Recall that an operator $T$ on a Hilbert space $\mathcal{H}$ is called essentially normal if its image in the Calkin algebra is normal or equivalently if the self-commutator $\left[T^{*}, T\right]=T^{*} T-T T^{*}$ is compact on $\mathcal{H}$.

In [8], the authors have determined which composition operators with automorphism symbols are essentially normal on $A^{2}\left(B_{N}\right)$ and $H^{2}\left(B_{N}\right)$ for $N \geq 1$. They have shown that the only essential normal automorphic composition operators are actually normal. This was first shown in the setting $H^{2}(\mathbb{U})$ by N. Zorboska in [17. The related works and some historical remarks can be found in [17, [9, 2] and [8]. In [3], the authors consider composition operators $C_{\varphi}$, where $\varphi$ is a linearfractional self-map of the unit disk $\mathbb{U}$, acting on the Dirichlet space $\mathcal{D}$. By using the E. Gallardo and A. Montes adjoint formula given in 6, they show that the essentially normal linear fractional composition operators on $\mathcal{D}$ are precisely those whose symbol is not a hyperbolic non-automorphism with a boundary fixed point. They also obtained conditions for the linear fractional symbols $\varphi$ and $\psi$ of the unit disk for which $C_{\psi}^{*} C_{\varphi}$ or $C_{\varphi} C_{\psi}^{*}$ is compact. As a result of our work we show that the operator $C_{\varphi}$ is essentially normal, when the symbol $\varphi$ is an automorphism.

In the next section, after giving some background material, by using the description of the adjoint of a linear-fractional composition operator acting on $\mathcal{D}$ given in [6], we give useful formulas for the operators $C_{\varphi}^{*} C_{\varphi}, C_{\varphi} C_{\varphi}^{*},\left[C_{\varphi}^{*}, C_{\varphi}\right]$ and $\left\{C_{\varphi}^{*}, C_{\varphi}\right\}$, when $\varphi$ is an automorphism. In section 3 we completely determine the point spectrum, spectrum and essential spectrum of $C_{\varphi}^{*} C_{\varphi}$ and $C_{\varphi} C_{\varphi}^{*}$. Finally in section 4 we determine the same for $\left[C_{\varphi}^{*}, C_{\varphi}\right]$ and $\left\{C_{\varphi}^{*}, C_{\varphi}\right\}$.

Our results and methods are quite different from those of 2, which pertain to the Hardy- and Bergman-space settings. For example, Theorem 3 of 2 shows that on the Hardy space $H^{2}(U)$, the self-commutator $\left[C_{\varphi}^{*}, C_{\varphi}\right]$ has no eigenvalues (given $\varphi(0) \neq 0$ ), while Theorem 4.1 below shows that on the Dirichlet space the spectrum of $\left[C_{\varphi}^{*}, C_{\varphi}\right]$ consists only of eigenvalues. Such differences are ultimately due to the fact that if $\varphi$ is an automorphism that does not fix 0 , then $C_{\varphi}^{*} C_{\varphi}$ and $C_{\varphi} C_{\varphi}^{*}$ are essentially Toeplitz operators with non-constant symbols in the Hardyspace setting; whereas, in the Dirichlet-space setting, these operators are essentially the identity.

\section{Preliminaries}

Throughout the paper for a Hilbert space $\mathcal{H}, \mathcal{B}(\mathcal{H})$ denotes the set of bounded operators on $\mathcal{H}$ and $\mathcal{B}_{0}(\mathcal{H})$ denotes the closed ideal of all compact operators in $\mathcal{B}(\mathcal{H})$. The natural homomorphism of $\mathcal{B}(\mathcal{H})$ onto the quotient Banach algebra $\mathcal{B}(\mathcal{H}) / \mathcal{B}_{0}(\mathcal{H})=\mathcal{B} / \mathcal{B}_{0}$ - the Calkin algebra-is denoted by $T \mapsto \widetilde{T}=T+\mathcal{B}_{0}(\mathcal{H})$. For an operator $T \in \mathcal{B}(\mathcal{H})$, the essential norm of $T$ is defined by

$$
\|T\|_{e}:=\inf \left\{\|T+K\|: K \in \mathcal{B}_{0}(\mathcal{H})\right\}
$$


and the essential spectrum $\sigma_{e}(T)$ is defined as the spectrum of the image $\widetilde{T}$ of $T$ in the Calkin algebra $\mathcal{B}(\mathcal{H}) / \mathcal{B}_{0}(\mathcal{H})$. It is well known that the essential spectrum of a normal operator consists of all points in the spectrum of the operator except the isolated eigenvalues of finite multiplicity (see page 359 of [4]).

As we mentioned in the Introduction, an operator $T$ on a Hilbert space $\mathcal{H}$ is called essentially normal if its image in the Calkin algebra is normal or equivalently if the self-commutator $\left[T^{*}, T\right]=T^{*} T-T T^{*}$ is compact on $\mathcal{H}$.

The Dirichlet space, which we denote by $\mathcal{D}$, is the set of all analytic functions $f$ on the unit disk $\mathbb{U}$ for which

$$
\int_{\mathbb{U}}\left|f^{\prime}(z)\right|^{2} d A(z)<\infty
$$

where $d A$ denote the normalized area measure. Equivalently an analytic function $f$ is in $\mathcal{D}$ if $\sum_{n=1}^{\infty} n|\widehat{f}(n)|^{2}<\infty$, where $\widehat{f}(n)$ denotes the $n$th Taylor coefficients of $f$. The inner product inducing the norm of $\mathcal{D}$ is given by

$$
\langle f, g\rangle_{\mathcal{D}}:=f(0) \overline{g(0)}+\int_{\mathbb{U}} f^{\prime}(z) \overline{g^{\prime}(z)} d A(z), \quad f, g \in \mathcal{D} .
$$

The inner product of two functions $f(z)=\sum_{n=0}^{\infty} \widehat{f}(n) z^{n}$ and $g(z)=\sum_{n=0}^{\infty} \widehat{g}(n) z^{n}$ in $\mathcal{D}$ may also be computed by

$$
\langle f, g\rangle_{\mathcal{D}}=\widehat{f}(0) \overline{\widehat{g}(0)}+\sum_{n=1}^{\infty} n \widehat{f}(n) \overline{\widehat{g}(n)} .
$$

The reproducing kernel consistent with the above product is given by

$$
K_{w}(z)=1+\log \frac{1}{1-\bar{w} z}=1+\sum_{n=1}^{k} \frac{\bar{w}^{n} z^{n}}{n}, w \in \mathbb{U} .
$$

For each holomorphic self-map $\varphi$ of $\mathbb{U}$ we define the composition operator $C_{\varphi}$ by the equation $C_{\varphi} f=f \circ \varphi(f \in \mathcal{D})$. A univalent self-map $\varphi$ of the unit disk is called a full map if it maps $\mathbb{U}$ onto its subset of full measure, i.e., $A(\mathbb{U} \backslash \varphi(\mathbb{U}))=0$.

It is shown in [10] that for any univalent full map $\varphi$,

$$
\left\|C_{\varphi}\right\|=\sqrt{\frac{L+2+\sqrt{L(4+L)}}{2}},
$$

where $L=-\log \left(1-|\varphi(0)|^{2}\right)$.

Let $\varphi$ be a linear fractional map of $\mathbb{U}$ defined by

$$
\varphi(z)=\frac{a z+b}{c z+d}
$$

such that $a d-b c \neq 0$ and $\varphi(\mathbb{U}) \subset \mathbb{U}$. Since linear fractional maps are univalent functions, they always induce bounded composition operators on the Dirichlet space. For such a linear fractional map define the associated linear fractional transformation $\varphi^{*}$ by

$$
\varphi^{*}(z)=\frac{\bar{a} z-\bar{c}}{-\bar{b} z+\bar{d}}
$$

The function $\varphi^{*}$ is a self-map of $\mathbb{U}$ because

$$
\varphi^{*}(z)=\frac{1}{\overline{\varphi^{-1}\left(\frac{1}{z}\right)}} .
$$


A conformal automorphism is a univalent holomorphic mapping of $\mathbb{U}$ onto itself. Each such map is linear fractional, and can be represented as a product $w \alpha_{p}$, where

$$
\alpha_{p}(z):=\frac{p-z}{1-\bar{p} z} \quad(z \in \mathbb{U})
$$

for some fixed $p \in \mathbb{U}$ and $w \in \partial \mathbb{U}$ (see [14]).

The results of this article are based on a formula, given by Gallardo-Gutierrez1 and Montes-Rodríguez in [6], for the adjoint of $C_{\varphi}$ on $\mathcal{D}$ when $\varphi$ is linear fractional. They have shown that $C_{\varphi}^{*}$ acting on the Dirichlet space is given by the formula

$$
C_{\varphi}^{*} f=f(0) K_{\varphi(0)}-\left(C_{\varphi^{*}} f\right)(0)+C_{\varphi^{*}} f, \quad f \in \mathcal{D} .
$$

By using this formula the operators $C_{\varphi}^{*} C_{\varphi}$ and $C_{\varphi} C_{\varphi}^{*}$ are identified as follows:

Theorem 2.1. Let $\varphi$ be a conformal automorphism on the unit disk $\mathbb{U}$. Then

$$
C_{\varphi}^{*} C_{\varphi}=I \bmod \mathcal{B}_{0}(\mathcal{D})
$$

and

$$
C_{\varphi} C_{\varphi}^{*}=I \bmod \mathcal{B}_{0}(\mathcal{D})
$$

where $\mathcal{B}_{0}(\mathcal{D})$ is the ideal of compact operators on $\mathcal{D}$.

Proof. By a simple computation and using formula (2.1) it follows that

$$
\left(C_{\varphi}^{*} C_{\varphi} f\right)(z)=f(\varphi(0)) K_{\varphi(0)}(z)-f\left(\varphi\left(\varphi^{*}(0)\right)\right)+f\left(\varphi\left(\varphi^{*}(z)\right)\right)
$$

and

$$
\left(C_{\varphi} C_{\varphi}^{*} f\right)(z)=f(0) K_{\varphi(0)}(\varphi(z))-f\left(\varphi^{*}(0)\right)+f\left(\varphi^{*}(\varphi(z))\right)
$$

for each $z \in \mathbb{U}, f \in \mathcal{D}$, and each linear fractional map $\varphi$. Now if $\varphi$ is a conformal automorphism, then $\varphi(z)=w \alpha_{p}(z)$, for some $p \in \mathbb{U}$ and $w \in \partial \mathbb{U}$. An easy calculation implies

$$
\varphi^{*}(z)=\frac{p-\bar{w} z}{1-\overline{w p} z}, \quad \varphi^{*} \circ \varphi(z)=z \text { and } \varphi \circ \varphi^{*}(z)=z .
$$

Hence for the conformal automorphism $\varphi$, the formulas (2.2) and (2.3) yield the following formulas:

$$
\left(C_{\varphi}^{*} C_{\varphi} f\right)(z)=f(\varphi(0)) K_{\varphi(0)}(z)-f(0)+f(z), \quad z \in \mathbb{U}, f \in \mathcal{D},
$$

$$
\left(C_{\varphi} C_{\varphi}^{*} f\right)(z)=f(0) K_{\varphi(0)}(\varphi(z))-f\left(\varphi^{*}(0)\right)+f(z), \quad z \in \mathbb{U}, f \in \mathcal{D} .
$$

Then by substituting $K_{\varphi(0)}(\varphi(z))=2+L-K_{p}(z)$ in formula (2.5), we obtain

$$
\left(C_{\varphi} C_{\varphi}^{*} f\right)(z)=f(z)-f(0) K_{p}(z)-f\left(\varphi^{*}(0)\right)+f(0)(2+L), z \in \mathbb{U}, f \in \mathcal{D} .
$$

Hence the operators $C_{\varphi}^{*} C_{\varphi}$ and $C_{\varphi} C_{\varphi}^{*}$ are finite rank perturbations of the identity.

As an immediate corollary of the above theorem we can identify the selfcommutator $\left[C_{\varphi}^{*}, C_{\varphi}\right]$ and anti-self-commutator $\left\{C_{\varphi}^{*}, C_{\varphi}\right\}$.

Corollary 2.2. Let $\varphi$ be a conformal automorphism on the unit disk $\mathbb{U}$. Then

$$
\left[C_{\varphi}^{*}, C_{\varphi}\right]=0 \bmod \mathcal{B}_{0}(\mathcal{D})
$$

and

$$
\left\{C_{\varphi}, C_{\varphi}^{*}\right\}=2 I \bmod \mathcal{B}_{0}(\mathcal{D})
$$

The first formula shows that $C_{\varphi}$ is essentially normal. 


\section{Spectrum of $C_{\varphi}^{*} C_{\varphi}$ And $C_{\varphi} C_{\varphi}^{*}$}

Let $\varphi$ be a conformal automorphism. In this section we are going to find the point spectrum, spectrum, essential spectrum and the eigenfunctions of the operators $C_{\varphi}^{*} C_{\varphi}$ and $C_{\varphi} C_{\varphi}^{*}$.

Theorem 3.1. Let $\varphi$ be a conformal automorphism on the unit disk $\mathbb{U}$ and $L=$ $-\log \left(1-|\varphi(0)|^{2}\right)$. Then

$$
\sigma_{e}\left(C_{\varphi}^{*} C_{\varphi}\right)=\{1\}=\sigma_{e}\left(C_{\varphi} C_{\varphi}^{*}\right)
$$

and

$$
\begin{aligned}
\sigma_{p}\left(C_{\varphi}^{*} C_{\varphi}\right) & =\sigma\left(C_{\varphi}^{*} C_{\varphi}\right) \\
& =\left\{1, \frac{L+2+\sqrt{L(4+L)}}{2}, \frac{L+2-\sqrt{L(4+L)}}{2}\right\} \\
& =\sigma_{p}\left(C_{\varphi} C_{\varphi}^{*}\right)=\sigma\left(C_{\varphi} C_{\varphi}^{*}\right) .
\end{aligned}
$$

Proof. Since the operators $C_{\varphi} C_{\varphi}^{*}$ and $C_{\varphi}^{*} C_{\varphi}$ are finite rank perturbations of the identity, the essential spectrum of each one is $\{1\}$. For the second part, since any points in the spectrum of a normal operator which are not in the essential spectrum are isolated eigenvalues of finite multiplicity, it is enough to find the eigenvalues. We do this first for the operator $C_{\varphi}^{*} C_{\varphi}$. Let $\lambda \in \mathbb{C}$ be an eigenvalue of the operator $C_{\varphi}^{*} C_{\varphi}$ with corresponding eigenvector $f \in \mathcal{D}$. Then $C_{\varphi}^{*} C_{\varphi} f=\lambda f$. By using formula (2.4) for $C_{\varphi}^{*} C_{\varphi}$, we have

$$
f(\varphi(0)) K_{\varphi(0)}(z)-f(0)+f(z)=\lambda f(z), \quad z \in \mathbb{U} .
$$

For the case $\lambda=1$, for each natural number $m$ and $n$, the function $z^{n}(z-\varphi(0))^{m}$ is a non-zero function in $\mathcal{D}$ that satisfies the equation and hence $\lambda_{0}=1$ is an eigenvalue of the operator $C_{\varphi}^{*} C_{\varphi}$ with infinite multiplicity. Now suppose that $\lambda \neq 1$. By putting $z=0$, it follows that $f(\varphi(0))=\lambda f(0)$ and

$$
\lambda f(0) K_{\varphi(0)}(z)-f(0)+f(z)=\lambda f(z) .
$$

By putting $z=\varphi(0)$, it follows that;

$$
\lambda f(0) K_{\varphi(0)}(\varphi(0))-f(0)+\lambda f(0)=\lambda^{2} f(0) .
$$

Since $\lambda \neq 0$ and $f$ is an eigenvector of the operator, $f(0) \neq 0$, and hence $\lambda$ must satisfy $\lambda^{2}-(2+L) \lambda+1=0$. Let $\lambda_{1}$ and $\lambda_{2}$ be the roots of this equation. By a simple computation, eigenfunctions of $C_{\varphi}^{*} C_{\varphi}$ are given by

$$
f_{i}(z)=1-\lambda_{i} K_{\varphi(0)}(z), \quad i=1,2,
$$

with corresponding eigenvalues $\lambda_{1}$ and $\lambda_{2}$. So

$$
\sigma_{p}\left(C_{\varphi}^{*} C_{\varphi}\right)=\sigma\left(C_{\varphi}^{*} C_{\varphi}\right)=\left\{1, \lambda_{1}, \lambda_{2}\right\}
$$

For the proof of other equalities, since the operator $C_{\varphi}$ is invertible, by using $C_{\varphi}$ 's polar decomposition, it follows that $C_{\varphi} C_{\varphi}^{*}$ is unitary equivalent to $C_{\varphi}^{*} C_{\varphi}$ and hence

$$
\sigma_{p}\left(C_{\varphi} C_{\varphi}^{*}\right)=\sigma\left(C_{\varphi} C_{\varphi}^{*}\right)=\left\{1, \lambda_{1}, \lambda_{2}\right\}
$$

Remark 3.2. The above theorem provides an alternative way to obtain the norm formula for an automorphic composition operator on $\mathcal{D}$. 


\section{The spectrum of $\left[C_{\varphi}^{*}, C_{\varphi}\right]$ And $\left\{C_{\varphi}^{*}, C_{\varphi}\right\}$}

Theorem 4.1. Let $\varphi=w \alpha_{p}$ be a conformal automorphism, for some fixed $p \in \mathbb{U}$ and $w \in \partial \mathbb{U}$, and let $M=-\log \left(1-\bar{w}|p|^{2}\right), L=-\log \left(1-|p|^{2}\right), r=2 L+M+L^{2}+\bar{M}$ and $s=L^{2}-M \bar{M}$. Then

$$
\sigma_{e}\left(\left[C_{\varphi}^{*}, C_{\varphi}\right]\right)=\{0\}
$$

and

$$
\sigma\left(\left[C_{\varphi}^{*}, C_{\varphi}\right]\right)=\sigma_{p}\left(\left[C_{\varphi}^{*}, C_{\varphi}\right]\right)=\left\{0, \lambda_{1}, \lambda_{2}, \lambda_{3}\right\},
$$

where $\lambda_{1}, \lambda_{2}$ and $\lambda_{3}$ are the roots of the cubic equation $\lambda^{3}-r \lambda+s=0$.

Proof. Let $T=C_{\varphi}^{*} C_{\varphi}-C_{\varphi} C_{\varphi}^{*}$. Then for each $f \in \mathcal{D}$ and $z \in \mathbb{U}$,

$$
(T f)(z)=f(\varphi(0)) K_{\varphi(0)}(z)+f(0) K_{p}(z)-f(0)(3+L)+f(p) .
$$

The first part is obtained by compactness of $T$. For the second part, since $T$ is compact, the non-zero points in the spectrum must be eigenvalues. Hence it is enough to find the eigenvalues of $T$. For this, let $\lambda$ be an eigenvalue of $T$. Then there is $f \in \mathcal{D}$ such that $T f=\lambda f$, i.e.,

$$
\lambda f(z)=f(\varphi(0)) K_{\varphi(0)}(z)+f(0) K_{p}(z)-f(0)(3+L)+f(p) .
$$

For the case $\lambda=0$, for each natural number $m, n$ and $k$, the function $z^{n}(z-\varphi(0))^{m}(z-p)^{k}$ is a nonzero function in $\mathcal{D}$ that satisfies equation (4.2) and hence $\lambda_{0}=0$ is an eigenvalue of the operator $T$ with infinite multiplicity.

Now suppose that $\lambda \neq 0$. We have three cases. First, assume that the values of $0, p, \varphi(0)$ are three distinct points. By putting $z=0, p, \varphi(0)$ in (4.2) we obtain the following system of equations:

$$
\left\{\begin{array}{l}
f(p w)+f(p)-(2+L+\lambda) f(0)=0 \\
f(p w)(1+M)+(1-\lambda) f(p)-2 f(0)=0 \\
f(p w)(1+L-\lambda)+f(p)-(2+L-\bar{M}) f(0)=0 .
\end{array}\right.
$$

This system of equations has a non-trivial solution only if the determinant of the coefficients is zero, i.e.,

$$
t(\lambda):=\operatorname{det}\left(\begin{array}{ccc}
1 & 1 & -(2+L+\lambda) \\
1+M & 1-\lambda & -2 \\
1+L-\lambda & 1 & -(2+L-\bar{M})
\end{array}\right)=\lambda^{3}-r \lambda+s=0,
$$

where $r=2 L+M+L^{2}+\bar{M}$ and $s=L^{2}-M \bar{M}$. In this case $w \neq 1$, and this cubic equation has three distinct real roots, $\lambda_{1}=2 \delta \cos \theta, \lambda_{2}=2 \delta \cos \left(\frac{2 \pi}{3}+\theta\right)$, and $\lambda_{3}=2 \delta \cos \left(\frac{4 \pi}{3}+\theta\right)$, where $\delta=\frac{\sqrt{3 r}}{3}, h=2 \delta^{3}$ and $\theta$ is chosen so that $\cos (3 \theta)=\frac{-s}{h}$ (see [12]).

Next, suppose $\varphi(0)=p$ and $p \neq 0$. Then $w=1$ and

$$
\lambda f(z)=(f(p)+f(0)) K_{p}(z)-f(0)(3+L)+f(p) .
$$

By putting $z=0, p$ in (4.3) we obtain the following system of equations:

$$
\left\{\begin{array}{l}
2 f(p)-(2+L+\lambda) f(0)=0 \\
(2+L-\lambda) f(p)-2 f(0)=0
\end{array}\right.
$$

This system of equations has a non-trivial solution only if $\lambda=\sqrt{L^{2}+4 L}$ or $\lambda=$ $-\sqrt{L^{2}+4 L}$.

Finally, suppose $\varphi(0)=p=0$. Then $(T f)(z)=0$ and $M=L=0$, and the results follows easily. 
By an easy calculation, it follows that for $i=1,2,3$ the function

$$
f(z)=\left(\lambda_{i}+\bar{M}\right)\left(K_{p w}(z)-1\right)+\left(\lambda_{i}-L\right)\left(K_{p}(z)+\lambda_{i}-1\right)
$$

is an eigenfunction corresponding to $\lambda_{i}$ when $\lambda_{i} \neq 0$.

Theorem 4.2. Let $w, p, \varphi, L$ and $M$ be as in Theorem 4.1. Then

$$
\sigma_{e}\left(\left\{C_{\varphi}^{*}, C_{\varphi}\right\}\right)=\{2\}
$$

and

$$
\sigma\left(\left\{C_{\varphi}^{*}, C_{\varphi}\right\}\right)=\sigma_{p}\left(\left\{C_{\varphi}^{*}, C_{\varphi}\right\}\right)=\left\{2, \lambda_{1}, \lambda_{2}, \lambda_{3}\right\},
$$

where $\lambda_{1}, \lambda_{2}$ and $\lambda_{3}$ are the roots of the cubic equation

$$
(\lambda-1)(\lambda-2-L)^{2}-(\lambda-2-M)(\lambda-2-\bar{M})=0 .
$$

Proof. Let $S=C_{\varphi}^{*} C_{\varphi}+C_{\varphi} C_{\varphi}^{*}$. Then for each $f \in \mathcal{D}$ and $z \in \mathbb{U}$,

$$
(S f)(z)=f(\varphi(0)) K_{\varphi(0)}(z)-f(0) K_{p}(z)+f(0)(1+L)-f(p)+2 f(z) .
$$

By Corollary 2.2, the operator $S$ is a compact perturbation of $2 I$, and hence the essential spectrum of $S$ is $\{2\}$. Also, since $S$ is self-adjoint, any points in the spectrum of $S$ which are not in the essential spectrum are eigenvalues of finite multiplicity. So it is enough to find such points. For natural numbers $m, n$ and $k$, the function $z^{n}(z-\varphi(0))^{m}(z-p)^{k}$ is an eigenfunction of $S$ with corresponding eigenvalue $\lambda=2$. Hence $\lambda_{0}=2$ is an eigenvalue of the operator $S$ with infinite multiplicity.

The other eigenvalues $\lambda$ of $S$ must be chosen such that

$$
h(\lambda):=(\lambda-1)(\lambda-2-L)^{2}-(\lambda-2-M)(\lambda-2-\bar{M})=0 .
$$

In the case that $w=1, h(\lambda)=(\lambda-2)(\lambda-2-L)^{2}$, and hence $\lambda_{1}=2$ and $\lambda_{2}=\lambda_{3}=$ $2+L$ are the roots of the equation. In that case the eigenfunction corresponding to $\lambda=L+2$ is

$$
f(z)=2+L-K_{\varphi(0)}(z)-K_{p}(z) .
$$

In other cases, since $h(1)=-|1+M|^{2}<0, h(2)=L^{2}-M \bar{M}>0$ and $h(L+2)=$ $-|L-M|^{2}<0$, it follows that the equation has three distinct roots $\lambda_{1}, \lambda_{2}$ and $\lambda_{3}$ such that

$$
1<\lambda_{1}<2<\lambda_{2}<L+2<\lambda_{3} .
$$

By simple computations, it follows that for $i=1,2,3$, the function

$$
f(z)=(\lambda-1)\left(2+L-\lambda_{i}\right) K_{p w}(z)-\left(2+M-\lambda_{i}\right) K_{p}(z)+\left(\lambda_{i}-1\right)(M-L)
$$

is an eigenfunction corresponding to $\lambda_{i}$.

Therefore

$$
\sigma(S)=\left\{2, \lambda_{1}, \lambda_{2}, \lambda_{3}\right\}
$$

where $\lambda_{1}, \lambda_{2}$ and $\lambda_{3}$ are the roots of the cubic equation

$$
(\lambda-1)(\lambda-2-L)^{2}-(\lambda-2-M)(\lambda-2-\bar{M})=0 .
$$

As a result of this section, we can determine the numerical range of $C_{\varphi}$, when $w=1$, i.e., when $C_{\varphi}$ is an involution. Recall that the numerical range of $C_{\varphi}$ is defined as

$$
W\left(C_{\varphi}\right):=\left\{\left\langle C_{\varphi} f, f\right\rangle: f \in \mathcal{D},\|f\|=1\right\} .
$$

Repeating the arguments of Proposition 5 of 2 we obtain the following corollary, which has the same result as before (for example see [13]). 
Corollary 4.3. Let $\varphi$ be an involution automorphism on the unit disk $\mathbb{U}$ and $L=$ $-\log \left(1-|\varphi(0)|^{2}\right)$. Then the closure of the numerical range of $C_{\varphi}$ is an ellipse with foci \pm 1 and with the major axis length $\sqrt{4+L}$.

\section{ACKNOWLEDGMENTS}

A substantial part of this paper was written at the University of Auckland during the author's sabbatical leave. The sabbatical at Auckland provided an ideal environment for working on this paper. The financial support during my sabbatical leave was provided by Shiraz University, and I would like to express my gratitude to them. I am also grateful to Professor Bill Barton and other colleagues in the mathematics department at the University of Auckland for their hospitality during this period. I wish to express my appreciation to my sisters and their families for their kindness and hospitality during my sabbatical leave. I would also like to thank the referee for useful and helpful comments and suggestions.

\section{REFERENCES}

1. A. Abdollahi, The numerical range of a composition operator with conformal automorphism symbol, Linear Algebra Appl. 408 (2005), 177-188. MR2166861(2006c:47027)

2. P. S. Bourdon and B. MacCluer, Selfcommutators of automorphic composition operators, Complex Variables and Elliptic Equations 52 (2007), 85-104. MR2275399

3. G. A. Chacón and G. R. Chacón, Some properties of composition operators on the Dirichlet space, Acta Math. Univ. Comenian. (N.S.) 74 (2005), no. 2, 259-272. MR 2195485 (2006i:47043)

4. J. B. Conway, A Course in Functional Analysis (II edition), Springer-Verlag, New York, 1990. MR.1070713 (91e:46001)

5. C. C. Cowen and B. D. MacCluer, Composition Operators on Spaces of Analytic Functions, CRC Press, Boca Raton, FL, 1995. MR1397026 (97i:47056)

6. Eva A. Gallardo-Gutierrez and Alfonso Montes-Rodríguez, Adjoints of linear fractional composition operators on the Dirichlet space, Math. Ann. 327 (2003), no. 1, 117-134. MR2005124 (2004h:47036)

7. C. Hammond, The norm of a composition operator with linear symbol acting on the Dirichlet space, J. Math. Anal. Appl. 303 (2005), no. 2, 499-508. MR2122233 (2006c:47028)

8. Barbara D. MacCluer and Matthew A. Pons, Automorphic composition operators on Hardy and Bergman spaces in the ball, Houston J. Math. 32 (2006), no. 4, 1121-1132. MR2268474

9. B. MacCluer and R. Weir, Essentially normal composition operators on Bergman spaces, Acta Sci. Math. (Szeged) 70 (2004), 799-817. MR.2107542 (2005i:47036)

10. M.J. Martin and D. Vukotic, Norms and spectral radii of composition operators acting on the Dirichlet spaces, J. Math. Analysis and Application 304 (2005), 22-32. MR2124646 (2006j:47043)

11. M.J. Martin and D. Vukotic, Adjoints of composition operators on Hilbert spaces of analytic functions, J. Funct. Anal. 238 (2006), no. 1, 298-312. MR2253017 (2007e:47035)

12. R.W.D. Nickalls, A new approach to solving the cubic: Cardan's solution revealed, The Mathematical Gazette 77 (1993), 354-359.

13. L. Rodman and I. M. Spitkovsky, On generalized numerical ranges of quadratic operators, http://arXiv.math.FA/0602318 v1 14 Feb 2006.

14. W. Rudin, Real and Complex Analysis. Third edition, McGraw-Hill Book Co., New York, 1987. MR.924157 (88k:00002)

15. J. H. Shapiro, Composition Operators and Classical Function Theory, Springer-Verlag, 1993. MR.1237406 (94k:47049) 
16. R. K. Singh and J. S. Manhas, Composition Operators on Function Spaces, North-Holland Publishing Co., Amsterdam, 1993. MR 1246562 (95d:47036)

17. N. Zorboska, Closed range essentially normal composition operators are normal, Acta Sci. Math. (Szeged) 65 (1999), 287-292. MR.1702203 (2000e:47048)

Department of Mathematics, Shiraz University, Shiraz 71454, Iran

E-mail address: abdolahi@shirazu.ac.ir 